\title{
The recovery of factors associated with decision- making capacity in individuals with psychosis
}

\author{
Colin Fernandez, Harry G. Kennedy and Miriam Kennedy
}

\section{Background}

There is limited data on the recovery of factors associated with decisional capacity in patients with psychosis.

\section{Aims}

To study the relationship between changes in mental capacity, symptoms and global functioning using structured measures during treatment for psychosis.

\section{Method}

Fifty-six patients with psychosis were assessed for capacity to consent to treatment on admission and at 6 and 12 weeks following treatment. The MacArthur Competence Assessment Tool - Treatment, the Positive and Negative Symptom Scale and the Global Assessment of Functioning Scale were used to measure mental capacities, symptom severity and global functioning respectively. Treating consultants rated capacity to consent, masked to these measures.

\section{Results}

Greater impairments on all measures were found in patients assessed as lacking capacity. These improved with treatment over 12 weeks with significant effect sizes (0.5 to 0.6). Stronger correlations between mental capacities, positive symptoms $(-0.47)$ and global functioning (0.56) were noted in the first 6 weeks.

\section{Conclusions}

Impairments in capacity in acute stages of psychosis are related to symptom severity and functional impairment. They improve during treatment, particularly in the first 6 weeks.

\section{Declaration of interest}

None.

\section{Copyright and usage}

(C) The Royal College of Psychiatrists 2017. This is an open access article distributed under the terms of the Creative Commons Non-Commercial, No Derivatives (CC BY-NC-ND) license.
Studies of treatment in mental illness should use measures of function as the outcome. Cognitive ability is likely to be central to this. ${ }^{1}$ There is already evidence that symptoms are related to neurocognitive changes ${ }^{2}$ and that neurocognitive impairments influence the behavioural handicaps of schizophrenia ${ }^{3}$ and treatment responses. ${ }^{4}$ Functional mental capacity is often used to refer to functional decision-making abilities required by a patient to make legally competent decisions about their care. ${ }^{5}$ Cross-sectional studies show that between $43.8 \%{ }^{6}$ and $60 \%{ }^{7}$ of patients admitted to a psychiatric hospital lack treatment-related functional decisional capacity at a given time. Up to $50 \%$ of patients admitted to hospital with an acute episode of schizophrenia and symptomatic bipolar disorder have impairments in at least one element of functional capacity to make decisions regarding treatment compared with between 20 and $25 \%$ of those admitted with depression..$^{8-10}$ Despite these figures, there are still relatively few studies of the recovery of functional mental capacity in patients being treated for psychosis. Although we know that evidence-based treatments such as antipsychotic medication can produce improvements in symptoms by 4-6 weeks, ${ }^{11}$ little is known about the rate of recovery of functional decision-making mental capacity in the early weeks of treatment or the relationship between capacity and changes in symptom severity or global functioning. Some research in forensic psychiatric populations addresses this issue and shows that patients' abilities to consent to treatment and to demonstrate fitness to plead do indeed improve with treatment; however, this has only been shown over long periods. ${ }^{12}$ In this population, different functional mental capacities were shown to correlate directly with global function and inversely with severity of psychotic symptoms. ${ }^{13}$ To date, no similar studies have been conducted in a non-forensic psychiatric population.

When the presence of incapacity is suspected, most jurisdictions would recommend employing a functional approach to assess this. ${ }^{14-17}$ The functional approach usually comprises tests of the ability to understand the relevant information, the ability to reason with the given information, the ability to appreciate consequences of any decision made and the ability to express a choice about the proposed treatment or intervention. ${ }^{18,19}$ Slight variability in these criteria does exist between jurisdictions, and for example, not all statutory or common law tests include 'appreciation'. However, 'appreciation' like 'believing' can be interpreted as an element of 'understanding' in that one has to believe that the information applies to their own personal situation in order to make a decision. ${ }^{20}$ With a functional approach, clinicians often use unstructured clinical methods of evaluating capacity and provide an opinion at the end on whether or not capacity is impaired. Although unstructured, clinical judgements are often guided by legal determinants of competency within a jurisdiction and still regarded as the gold standard in determining capacity. ${ }^{21}$

In research, however, more structured assessments are usually used, and the MacArthur Competence Assessment Tool Treatment (MacCAT-T) is the most widely accepted tool for assessment of functional mental capacity. ${ }^{22}$ The MacCAT-T tool was developed through a long history of dialogue between psychiatrists acting as expert witnesses and judges guiding how laws are to be applied. It was based on research findings that compared components of decisional abilities of healthy controls with newly admitted patients who were medically ill, or who had depression or schizophrenia. ${ }^{23-25}$ It focuses on four decisional abilities (understanding, reasoning, appreciation and ability to express a choice) that, although not exactly, do share a good degree of overlap with most legal tenets of competence across jurisdictions.

In this study, we aimed to determine the prevalence of incapacity to give consent to treatment as determined by the treating consultant psychiatrists using unstructured clinical opinion in patients with a psychosis at the point of admission to hospital and then at 6 and 12 weeks following treatment. We used the MacCAT-T to track changes in measures of decisional abilities 
along with measures of symptom severity and measures of global functioning at each of these time points. We aimed to compare changes in the clinicians' rating of the presence or absence of capacity with MacCAT-T measures of functional decisional abilities, symptom severity and global functioning. We hypothesised that clinically assessed capacity status would improve in parallel with MacCAT-T measures, symptoms and global function. In Ireland, where this study was conducted, clinicians are recommended to employ a functional approach in their assessments of capacity based on the necessary decisional abilities required for the task at hand. ${ }^{26}$ This is further reflected in the recently published Assisted Decision-Making (Capacity) Act 2015 that aims to bring Irish legislation in this field up to standards set out by the United Nations Convention on the Rights of Persons with Disabilities (UNCRPD). ${ }^{27}$

\section{Method}

The study was conducted in a general adult psychiatric hospital in Dublin, Ireland. The study protocol was approved by the hospital research and ethics committee before its commencement. All participants were aged between 18 and 65 and recruited from an in-patient psychiatric intensive care unit. All participants in the study met the DSM-IV-TR criteria ${ }^{28}$ for a psychotic disorder (schizophrenia, major depressive disorder with psychotic features, psychotic disorder because of psychoactive substance misuse, schizoaffective disorder, psychosis in bipolar disorder and psychotic disorder not otherwise specified). Informed consent to participate in the study was obtained from participants. Exclusion criteria included a primary diagnosis of delirium, dementia or intellectual disability (moderate/severe), other cognitive disorders or inadequate understanding of the English language. Assessments were conducted at three time points during the study: the first baseline assessment within $48 \mathrm{~h}$ of admission, the second at 6 weeks and finally the third at 12 weeks. Each patient had an individual care plan, and treatment was in keeping with National Institute for Health and Care Excellence (NICE) guidelines for schizophrenia, ${ }^{11}$ major depression ${ }^{29}$ or bipolar disorder ${ }^{30}$ as appropriate that included antipsychotic medication, nursing and medical care, psychoeducation, relapse prevention and a recovery-oriented approach.

\section{Assessments}

All assessments were carried out by the first author. Assessments were carried out on participants at each of the three time points as described above and consisted of a semi-structured interview with completion of the MacCAT-T, the Positive and Negative Symptom Scale (PANSS) and the Global Assessment of Functioning Scale (GAF). The treating consultant was requested to record their opinion regarding each patient's capacity to consent to treatment with an antipsychotic at each of the three time points. This was an unstructured clinical opinion based on guidelines set out by the Irish Medical Council that recommends a functional approach focusing on a patient's ability to understand, retain, use or weigh up the relevant information they have been given and to communicate a choice about treatment. ${ }^{26}$ This opinion was independent of the research assessments and masked to those assessments.

The MacCAT-T comprises four subscales that measure the four abilities involved in decision-making: understanding, reasoning, appreciation and the ability to express a choice. ${ }^{5}$ A sum of these subscales is recorded as a total score at the end. The MacCAT-T Understanding subscale measures a patient's comprehension of the information given to them regarding the proposed treatment and has a score range from 0 to 6 . The Reasoning subscale provides a measure of the patient's ability to reason with the information and their ability to generate consequences for each decision they may take and has a range from 0 to 8 . The Appreciation subscale has a score range from 0 to 4 and measures the degree to which a patient appreciates and understands how the information about the proposed treatment applies to them and their current circumstances. The Choice subscale measures the patient's ability to express a choice regarding the proposed treatments and has a range from 0 to 2 . The MacCAT-T total score has a range from 0 to 20 .

The PANSS is a validated medical scale used to measure the severity of symptoms of schizophrenia. ${ }^{31}$ It offers both a total score and subscale scores for positive, negative and general symptoms exhibited during an episode of psychosis. The GAF is a scale that measures the social, occupational and psychological functioning of adults. ${ }^{28}$

\section{Statistical analysis}

All results were analysed and calculated using IBM SPSS Statistics Version 20. Non-parametric tests used were the Friedman test, Wilcoxon signed rank test and Spearman's rank correlation coefficient to avoid assumptions regarding the distribution of variables obtained from the structured assessments. Effect sizes, $r$, were calculated to compare changes between measures of functional decisional abilities, symptom severity measures and global functioning measures at each of the three time points of assessment. Binary logistic regression was used to further assess the relationship between MacCAT-T scores, PANSS scores and the clinicians' assessment of capacity to give or withhold consent.

\section{Ethical issues}

As this is a study focusing on capacity to consent to treatment in patients with psychosis, the study material and structured assessments focused solely on the functional ability to give consent to treatment and any incapacity discovered in this regard did not automatically imply that the patient lacked the specific competencies to give consent to participate in the study. Consent to participate in this research project represented a lesser cognitive burden than consent to treatment with an antipsychotic as assessed in the research protocol itself. Accordingly, the group of patients who consented to participate was expected to include patients who both had and did not have capacity to consent to treatment as assessed by the research protocol. This is in accordance with other research on capacity to consent to participating in research. ${ }^{32,33}$ It is worth emphasising that all patients recruited into the study gave informed consent to participate in the study and the study protocol received ethical approval from the hospital research and ethics committee before participant recruitment. Signed consent to participate in the study was sought and obtained from each participant at the start of the study. Consent to continue participation in the study was obtained at each of the following two time points (at 6 and 12 weeks) during the 12 -week period. Participants were given the option to withdraw from the study at any point. Each participant was given written information regarding the purpose and nature of the study including the anonymous nature of data collection and confidentiality. Those who withdrew consent on follow-up were not included in data analysis at any time period. Patients were given the opportunity to ask any questions they had regarding the study. In effect, the assessments carried out on patients in the study were merely a more structured form of routine assessments that would have normally been conducted by their respective treating teams while in hospital.

\section{Sample characteristics}

A total of 91 patients met inclusion criteria (Fig. 1) but 12 declined to participate and could not be assessed further. In total, 


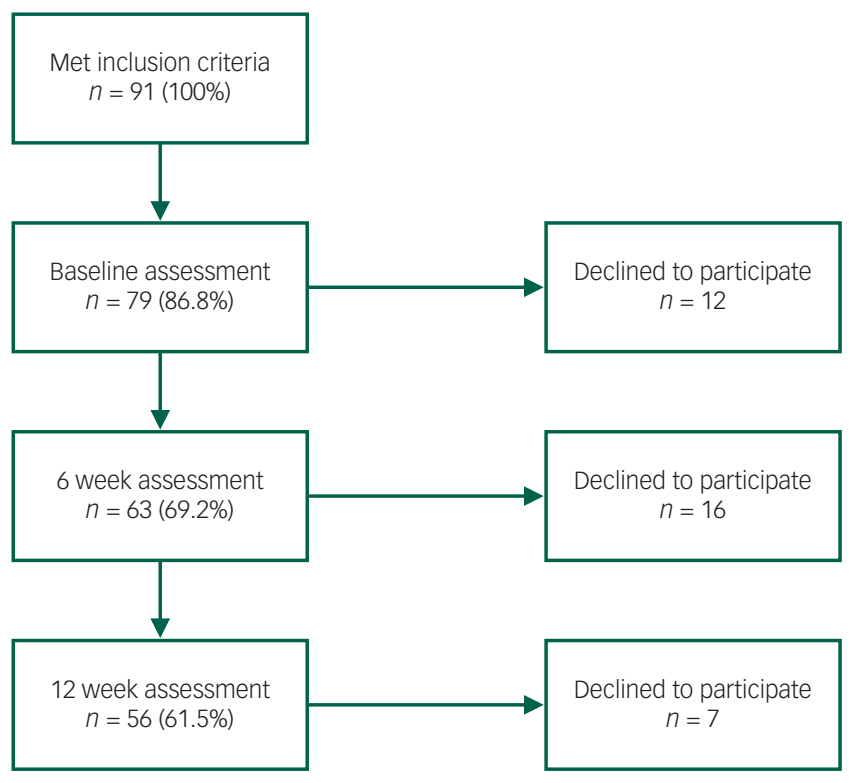

Fig. 1 Study recruitment diagram.

79 patients consented to the initial baseline assessment. At 6 weeks, 16 participants withdrew consent to continue to participate further in the study, and at 12 weeks a further 7 participants withdrew their consent, leaving a total of 56 participants who completed the full 12-week duration of the study. All 23 patients who withdrew their consent during the study were out-patients and did not wish to return for further assessment. Of the 56 patients who completed the study, 52 patients remained as inpatients in the hospital for the entire 12-week duration of the study, and 4 patients were out-patients at the 12 -week point. This was a $61.6 \%$ retention rate of the original sample. The mean age of participants was 38.2 years (s.d.=12). There was an almost equal gender distribution with 29 (51.8\%) male and 27 (48.2\%) female participants. Twenty-nine patients were diagnosed with schizophrenia $(n=29)$, ten with a psychosis in bipolar disorder $(n=10)$, seven with major depressive episode and psychotic features $(n=7)$, seven with schizoaffective disorder $(n=7)$, two with psychotic disorder because of psychoactive substance misuse $(n=2)$ and one with a psychotic disorder not otherwise specified $(n=1)$.

\section{Results}

In Table 1, at the point of admission, 21 (37.5\%) participants were found to lack capacity to give consent to treatment based on the individual treating clinicians' assessment. This figure dropped to $10(17.9 \%)$ participants at 6 weeks and $3(5.4 \%)$ participants by 12 weeks following treatment. Measures on the MacCAT-T mean scores show significantly lower total scores in the population of participants deemed to be lacking capacity when compared with the participants who did not show the presence of incapacity. The MacCAT-T subscales for understanding, reasoning, appreciation and choice all show significantly lower mean values in the population lacking capacity. Symptom severity measures on the PANSS and the degree of functional impairment measured by the GAF all showed significantly greater levels of impairments in the population lacking capacity.

Table 2 shows that at baseline, the mean MacCAT-T total score and all MacCAT-T subscales show low mean values (all less than half of the total scores possible) indicating that the baseline population had significantly impaired decisional abilities in all

\begin{tabular}{|c|c|c|c|c|c|c|c|c|c|c|c|c|}
\hline \multirow[b]{3}{*}{ linician rating of capacity } & \multicolumn{3}{|c|}{ No incapacity } & \multicolumn{3}{|c|}{ Incapacity present } & \multicolumn{6}{|c|}{ Mann-Whitney $U$ test } \\
\hline & \multirow[b]{2}{*}{ Baseline } & \multirow[b]{2}{*}{6 weeks } & \multirow[b]{2}{*}{12 weeks } & \multirow[b]{2}{*}{ Baseline } & \multirow[b]{2}{*}{6 weeks } & \multirow[b]{2}{*}{12 weeks } & \multicolumn{2}{|c|}{ Baseline } & \multicolumn{2}{|c|}{6 weeks } & \multicolumn{2}{|c|}{12 weeks } \\
\hline & & & & & & & Z & $P$ & Z & $P$ & Z & $P$ \\
\hline Number of patients & 35 & 46 & 53 & 21 & 10 & 3 & & & & & & \\
\hline \multicolumn{13}{|l|}{ MacCAT-T } \\
\hline Understanding & 4.2 & 5.3 & 5.9 & 1.4 & 2.9 & 3.0 & -5.0 & 0.001 & -4.5 & 0.001 & -4.1 & 0.001 \\
\hline Reasoning & 3.9 & 6.0 & 7.4 & 0.3 & 2.0 & 1.7 & -5.3 & 0.001 & -4.2 & 0.001 & -3.6 & 0.001 \\
\hline Appreciation & 1.4 & 2.8 & 3.5 & 0.0 & 0.6 & 0.3 & -4.5 & 0.001 & -4.1 & 0.001 & -3.6 & 0.001 \\
\hline Choice & 1.4 & 1.9 & 2.0 & 0.6 & 1.4 & 1.7 & -3.0 & 0.003 & -2.3 & 0.019 & -4.2 & 0.001 \\
\hline Total & 10.8 & 16.0 & 18.8 & 2.4 & 6.9 & 7.4 & -5.2 & 0.001 & -4.4 & 0.001 & -3.2 & 0.001 \\
\hline \multicolumn{13}{|l|}{ PANSS } \\
\hline Positive & 20.0 & 12.7 & 11.7 & 27.7 & 25.7 & 23.7 & -3.6 & 0.001 & -4.7 & 0.001 & -2.9 & 0.004 \\
\hline Negative & 18.1 & 14.2 & 10.8 & 24.1 & 21.6 & 19.7 & -2.5 & 0.013 & -2.9 & 0.004 & -2.3 & 0.021 \\
\hline General & 41.5 & 30.7 & 23.0 & 54.5 & 49.0 & 43.0 & -3.4 & 0.001 & -4.1 & 0.001 & -2.8 & 0.005 \\
\hline Total & 79.3 & 58.6 & 42.8 & 106.3 & 96.3 & 86.3 & -4.0 & 0.001 & -4.1 & 0.001 & -2.9 & 0.004 \\
\hline GAF & 36.2 & 56.2 & 67.4 & 27.0 & 34.2 & 36.0 & -3.9 & 0.001 & -4.7 & 0.001 & -2.7 & 0.007 \\
\hline
\end{tabular}




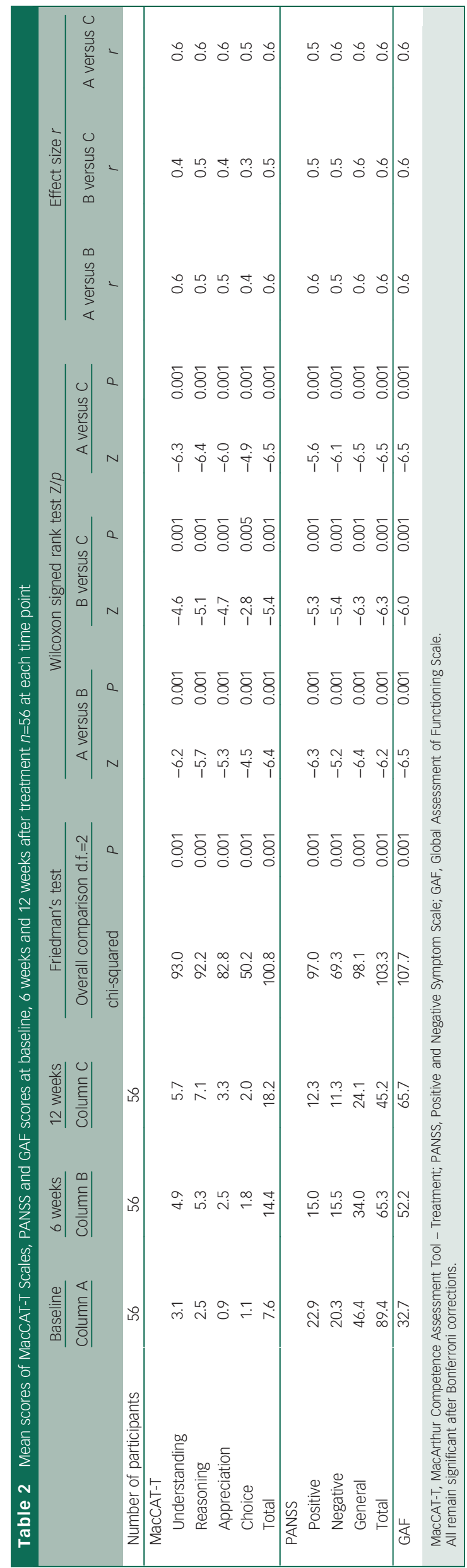

domains. These improved significantly over the course of the study, and by 6 weeks, all subscales showed more than half of the totals possible. By 12 weeks, all MacCAT-T subscales were either fully or nearly restored to their total values. The exception to this was with the ability to express a choice subscale that was almost fully restored by 6 weeks and fully restored by 12 weeks. Table 2 also shows that measures of understanding, appreciation, choice and total scores improved with larger effect sizes in the first 6 weeks compared with effect sizes for the same measures in the second 6 weeks. Both the PANSS and GAF also improved in a significant manner at each time point with effect sizes of 0.5 to 0.6 .

Table 3 shows that increase in the mean MacCAT-T total scores and reduction in the mean total PANSS scores show a strong correlation value of -0.67 over the entire 12 -week duration of the study. In the first 6 weeks, a medium correlation value of -0.39 was observed between the change in MacCAT-T total scores and mean total PANSS scores. From week 6 to week 12, this value was greater at -0.59 . In the first 6 weeks, reductions in positive symptoms correlated strongly with improvements in the MacCAT-T total scores $(-0.47)$ compared with reductions in negative or general symptom scales. Increases in GAF scale measures, as well, show stronger correlations with improvements in the MacCAT-T total scores in the first 6 weeks compared with the weeks after.

To assess which variables most closely corresponded to the treating clinicians' assessment of capacity to give or withhold consent, we used binary logistic regression. The outcome was change from incapacity present to no incapacity present over the course of the 12-week period of observation. Variables entered were change in MacCAT-T total score, change in PANSS total score and change in GAF score. The model correctly classified $76.8 \%$ of cases, with an omnibus test $X^{2}=23.4$, d.f. $=3, P<0.001$; Nagelkerke's $R^{2}=0.478$ and Hosmer and Lemshow $X^{2}=6.2$, d.f. $=7$, $P=0.514$. Only change in MacCAT-T total score had a significant odds ratio $(\mathrm{OR})=1.351,95 \%$ CI $1.110-1.645, P=0.003$. To check for a possible effect of symptoms within the PANSS subscales, a model was constructed using only the PANSS subscales. This model did not have a significant omnibus test, Nagelkerke's $R^{2}=0.154$, and although $75 \%$ of cases appeared to be correctly classified, no PANSS subscale had a significant odds ratio. We then examined which of the MacCAT-T subscales best approximated the clinicians' judgement of regaining capacity. This model had an omnibus test of $X^{2}=27.4$, d.f. $=4, P=0.001$, Nagelkerke's $R^{2}=0.541$, Hosmer and Lemshow $X^{2}=6.8$, d.f. $=7, P=0.452$, and $83.9 \%$ of cases were correctly classified. For understanding: $\mathrm{OR}=$ 2.875, 95\% CI 1.474-5.609, $P=0.002$; reasoning: $\mathrm{OR}=1.422,95 \% \mathrm{CI}$ $0.882-2.293, P=0.148$; appreciation: $\mathrm{OR}=1.196,95 \%$ CI $0.619-$ 2.309, $P=0.594$; expressing a choice: $\mathrm{OR}=0.371,95 \%$ CI $0.117-$ $1.182, P=0.094$.

\section{Discussion}

\section{Main findings}

At the point of admission to hospital, a significant number of patients with a psychosis showed the presence of incapacity to give consent to treatment as determined by their individual treating consultants. This figure dropped significantly by 6 weeks and 12 weeks. All measures of decisional abilities on the MacCAT-T were significantly lower in the population of patients showing incapacity when compared with the population with no incapacity. Grisso \& Appelbaum highlight that although measures on the MacCAT-T do not offer an arbitrary threshold score for determining the presence of incapacity to make decisions about treatment, scores that are below the average range for the four respective decision-making abilities do indicate severely affected 


\begin{tabular}{|c|c|c|c|c|c|c|}
\hline \multirow[b]{2}{*}{ Change in scores } & \multicolumn{2}{|c|}{ From baseline to week 6} & \multicolumn{2}{|c|}{ From week 6 to week 12} & \multicolumn{2}{|c|}{ From baseline to week 12} \\
\hline & GAF & MacCAT-T total & GAF & MacCAT-T total & GAF & MacCAT-T total \\
\hline \multicolumn{7}{|l|}{ PANSS } \\
\hline Positive & -0.50 & -0.47 & -0.26 & -0.46 & -0.32 & -0.23 \\
\hline Negative & -0.05 & -0.32 & -0.09 & -0.31 & -0.09 & -0.50 \\
\hline General & -0.34 & -0.26 & -0.24 & -0.56 & -0.18 & -0.54 \\
\hline Total & -0.39 & -0.39 & -0.22 & -0.59 & -0.17 & -0.67 \\
\hline GAF & & 0.46 & & 0.33 & & 0.15 \\
\hline
\end{tabular}

abilities in these domains that would likely demonstrate an incapacity to give valid consent. ${ }^{5}$ Our findings corroborate this. The population of patients assessed as lacking capacity showed a greater degree of symptom severity and impairment of global functioning. All measures of decisional abilities, symptom severity and global function improved significantly within 6 weeks and again by 12 weeks. Further improvements may have been observed beyond this had measurements continued. Improvement in a patient's decision-making skills as their symptoms decrease is a phenomenon that is often observed by clinicians in clinical practice but has limited evidence in the form of corroboration from research measures such as the MacCAT-T which our study offers. Measures of understanding, reasoning and appreciation improved in a similar manner throughout the 12 weeks but at a quicker rate in the first 6 weeks and were almost fully restored by 12 weeks. The ability to express a choice appeared to be fully restored by 6 weeks in most but not all patients, indicating that in some patients this may reflect the expression of choices that were not based on intact capacities at earlier points of recovery.

The improvements in measures of capacity correlated strongly with measures of the severity of symptoms (PANSS) and measures of functional impairment (GAF) indicating that impairments in capacity are related and likely to be proportional to the severity of symptoms and the degree of functional impairment. At baseline, the mean PANSS total score of 89.4 corresponded to between the 'moderate' to 'markedly ill' range on the Clinical Global Impressions (CGI) Scale. ${ }^{34}$ This decreased to 65.3 ('mild' to 'markedly ill' range on the CGI) at 6 weeks and then to 45.2 (below the 'mildly ill' range) at 12 weeks. The mean GAF scores on initial assessment (32.7) corresponded to the 'seriously impaired' function range and at 6 weeks improved to the 'moderately impaired' function range (52.2) and by 12 weeks was within the 'mild impairment' of function (65.7). The percentage reduction of the mean PANSS total scores in our study from baseline measures was $26.9 \%$ at 6 weeks and $49.4 \%$ at 12 weeks which is comparable to the recommended $50 \%$ reduction from baseline cut-off values to constitute treatment response in most published drug trials. ${ }^{34}$ We found stronger correlations between positive symptoms on the PANSS with improvements in measures of functional capacity on the MacCAT-T in the early stages of treatment. This finding is supported by Howe et $a l^{35}$ who showed that elevated scores on positive, cognitive and excitement in the PANSS factor scores had lower MacCAT-T scores.

Binary logistic regression showed that the factor best describing the clinical assessment that patients who had lacked capacity had become competent was the MacCAT-T assessment. Neither change in symptom severity nor change in global function significantly contributed to a statistical model of this change in competence. However, change in the MacCAT-T assessment instrument was statistically a good model of how clinicians assessed change in capacity. Within the MacCAT-T scales, change in the measure of understanding appeared to be the best model for how clinicians made this decision. This may suggest that clinicians were either too narrowly focused on this cognitive domain or that clinicians are not sufficiently aware of the complementary domains of reasoning, appreciation and ability to express a choice.

When compared with similar studies conducted in a forensic psychiatric population, the trend of results in this study is in keeping with their findings that measures of different functional mental capacities do correlate directly with global function and inversely with scores for severity of psychosis. ${ }^{13}$ Also in forensic populations consisting of longer-term patients, functional mental capacities for consent to treatment and fitness to plead have been shown to improve over time with treatment as usual, with effect sizes between 0.3 and $0.7^{12}$ which are comparable to results in this study. A few cross-sectional studies offer conflicting results from ours and argue that impairments in decisional competencies are only modestly related to psychotic symptoms and are more strongly related to cognitive dysfunction. ${ }^{36}$ In middle-aged or older populations of patients with schizophrenia, cross-sectional associations between cognitive dysfunction and capacity to consent rather than symptom severity have been shown to be true and encourage clinicians to develop more effective methods of providing informed consent in this population. ${ }^{37}$ Another crosssectional study demonstrated that in a population of patients with chronic psychosis, decisional competencies to consent to treatment were more related to cognitive and negative symptoms than the classic positive symptoms. ${ }^{38}$ A recently published study in a forensic population adds that neurocognitive impairment mediates some effects of symptoms on decisional capacity. ${ }^{39}$ These studies probably reflect the degree of heterogeneity within a psychiatric population, and consideration should be given to other factors within subsets of the population such as age-related cognitive decline when assessing capacity.

\section{Study limitations}

The main limitation of this study was the relatively small population size of 56 patients. The sample studied was diagnostically heterogeneous with a high number of affective psychoses rather than solely schizophrenia, and this may limit the association of results to one specific diagnostic group. Selection and attrition biases may also be considered as potential limitations as during the stage of recruiting participants, 91 patients met the inclusion criteria but 12 declined to participate initially and 23 subsequently withdrew their consent to continue in the study and therefore could not be assessed further or included in the analysis.

In the study, patients demonstrated improvements in capacity to consent to treatment with an antipsychotic that correlated with improvements in measures of decisional abilities, symptom severity and global functioning while undergoing treatment largely in an in-patient setting. The treatment offered to patients in the hospital consisted of individual care plans as per NICE guidelines 
and included antipsychotic medications along with nursing care, psychoeducation, relapse prevention strategies and a recoveryoriented approach. It was not possible to rigorously standardise the treatments delivered to each patient. The study did not take into account the potential for slight variability in each treatment plan such as types of antipsychotics used or combinations of treatments used. More variability may have occurred in the later stages as patients availed of periods of temporary leave from hospital and transitioned to out-patient care. Treatment in the study should best be regarded as 'treatment as usual' and would have a degree of standardisation with other jurisdictions based on its adherence to the NICE guidelines. All patients who remained as in-patients for the duration of the 12-week study were reported to be compliant with antipsychotic medication but this could not be ensured during periods of leave from hospital or as out-patients. Given the additional non-pharmacological interventions in individual care plans whose effects were not controlled for, it is not possible to relate the improvements in capacity to antipsychotic medication alone. It was also possible that some of these patients may have simply improved because of the natural course of their illness, and treatments received may or may not have had any significant effect on their symptoms or decisional abilities.

Similarly, it was not possible to rigorously structure or standardise the assessments by individual clinicians of the presence or absence of capacity. Sixteen different clinicians treated patients in the study. Although this may be considered unreliable by some, it was probably an accurate depiction of how capacity is currently assessed clinically in most jurisdictions as clinicians in Ireland and in other similar jurisdictions assess capacity using a functional approach based on the criteria as discussed in the introduction.

\section{Clinical implications}

Based on our findings that impairments in functional capacity to give consent to treatment in patients with psychosis improved with the reduction of symptoms and improvement of global function, we would recommend that clinicians utilise treatments that focus on the reduction of psychotic symptoms in the weeks after admission. We found the reduction in positive symptoms in the early weeks correlated strongly with improvements in decision-making abilities, and thus, treatments that address this are likely to be beneficial. Similarly, increases in levels of global function as depicted by the GAF scores correlated with decisionmaking abilities; hence, interventions that aim to improve global levels of function are likely to be of value in further improving functional mental capacities.

Given the significant proportion of patients deemed to be lacking in capacity to consent to treatment at the point of admission, we recommend that clinicians be aware of this when treating patients in the acute phase of psychosis. Using an instrument such as the MacCAT-T in routine practice may be considered as an aid or guide to decision-making. For example, clinicians may currently be influenced by deficits in understanding as shown here, but may not be sufficiently aware of impairments in reasoning as shown by the ability to appreciate the consequences of the choices available and to compare choices accordingly. There is, however, no indication that structured instruments such as the MacCAT-T should be used as 'actuarial' determinants of fixed threshold scores for capacity or incapacity. In the alternative, training should focus on the assessment of the domains of understanding, reasoning (consequential and comparative), appreciation and ability to express a choice.

The PANSS and GAF may be of additional value in helping to support a clinical judgement of incapacity given our findings that show direct and inverse correlations, respectively, between capacity and these measures. Our findings also indicate that the presence of incapacity in the acute stages of psychosis is not a static state and does improve in a significant proportion of patients admitted to hospital as quickly as in 6 weeks with further improvements observed in the weeks after.

\section{Legal implications}

This study was conducted in Ireland where mental health legislation in the form of the Mental Health Act 2001 allows for the treatment of involuntary patients who cannot give valid consent for up to 3 months before an assessment of their capacity to consent to treatment is required to determine whether treatment without consent is still necessary (Section 60 of the Mental Health Act 2001). In keeping with findings from this study, there may be evidence that the duration of 3 months is excessive, and earlier reviews of capacity to consent to treatment would be more in keeping with respecting a person's human rights.

Colin Fernandez, MSC, MRCPsych, St Patrick's University Hospital, Dublin Ireland; Harry G. Kennedy, MD, FRCPsych, Academic Department of Psychiatry, University of Dublin, Trinity College, Dublin, Ireland; National Forensic Mental Health Service, Central Mental Hospital, Dublin, Ireland; Miriam Kennedy, PhD, MRCPsych, St Patrick's University Hospital, Dublin, Ireland; Academic Department of Psychiatry, University of Dublin, Trinity College, Dublin, Ireland

Correspondence: Professor Harry G. Kennedy, Central Mental Hospital, Dundrum, Dublin 14, Ireland. Email: kennedh@tcd.ie

First received 20 Oct 2016, final revision 24 Mar 2017, accepted 27 Mar 2017

\section{References}

1 Kahn RS, Keefe RS. Schizophrenia is a cognitive illness: time for a change in focus. JAMA PSychiatry 2013; 70: 1107-12.

2 Anda L, Brønnick KS, Johnsen E, Kroken RA, Jørgensen H, Løberg EM. The course of neurocognitive changes in acute psychosis: relation to symptomatic improvement. PLOS One 2016; 11: e0167390.

3 O'Reilly K, Donohoe G, Coyle C, O'Sullivan D, Rowe A, Losty M, et al. Prospective cohort study of the relationship between neuro-cognition, social cognition and violence in forensic patients with schizophrenia and schizoaffective disorder. BMC Psychiatry 2015; 15: 155.

4 O'Reilly K, O'Connell P, Donohoe G, Coyle C, O'Sullivan D, Azvee Z, et al. Anticholinergic burden in schizophrenia and ability to benefit from psychosocial treatment programmes: a 3-year prospective cohort study. Psychol Med 2016; 46: 3199-211.

5 Grisso T, Appelbaum PS. Assessing Competence to Consent to Treatment. Oxford University Press, 1998.

6 Cairns R, Maddock C, Bucahanan A, David AS, Hayward P, Richardson G, et al. Prevalence and predictors of mental incapacity in psychiatric in-patients. $\mathrm{Br} J$ Psychiatry 2005; 187: 379-85.

7 Owen G, Richardson G, David AS, Szmukler G, Hayward P, Hotopf M. Mental capacity to make decisions on treatment in people admitted to psychiatric hospitals: cross sectional study. Br Med J 2008; 337: a448.

8 Grisso T, Appelbaum PS. The MacArthur Treatment Competence Study. III: abilities of patients to consent to psychiatric and medical treatments. Law Hum Behav 1995; 19: 149-74.

9 Vollmann J, Bauer A, Danker-Hopfe H, Helmchen H. Competence of mentally ill patients: a comparative empirical study. Psychol Med 2003; 33: 1463-71.

10 Palmer BW, Dunn LB, Depp CA, Eyler LT, Jeste DV. Decisional capacity to consent to research among patients with bipolar disorder: comparison with schizophrenia patients and healthy subjects. J Clin Psychiatry 2007; 68: 689-96.

11 National Institute for Health and Care Excellence. Psychosis and Schizophrenia in Adults: Prevention and Management. NICE, 2014 (https://www.nice.org.uk/ guidance/cg178).

12 Dornan J, Kennedy M, Garland J, Rutledge E, Kennedy HG. Functional mental capacity, treatment as usual and time: magnitude of change in secure hospital patients with major mental illness. BMC Res Notes 2015; 8: 566.

13 Rutledge $\mathrm{E}$, Kennedy M, Neill HO, Kennedy HG. Functional mental capacity is not independent of the severity of psychosis. Int J Law Psychiatry 2008; 31: 9-18.

14 Australian Law Reform Commission. Informed Decisions about Medical Procedures. ALRC, 1989. 
15 Law Commission. Mental Incapacity. HMSO, 1995.

16 Law Reform Commission Ireland. Vulnerable Adults and the Law. LRC, 2006.

17 Ryan C, Callaghan S, Peisah C. The capacity to refuse psychiatric treatment: a guide to the law for clinicians and tribunal members. Aust N Z J Psychiatry 2015 49: 324-33.

18 Appelbaum PS. Assessment of patients' competence to consent to treatment N Engl J Med 2007; 357: 1834-40.

19 Berg JW, Appelbaum PS, Grisso T. Constructing competence: formulating standards of legal competence to make medical decisions. Rutgers Law Rev 1996; 48: 345-96.

20 Fitzpatrick \& Anor v. K. \& Anor (2008) IEHC 104

21 Moye J, Gurrera RJ, Karel MJ, Edelstein B, O'Connell C. Empirical advances in the assessment of the capacity to consent to medical treatment: clinical implications and research needs. Clin Psychol Rev 2006; 26: 1054-77.

22 Dunn LB, Nowrangi MA, Palmer BW, Jeste DV, Saks ER. Assessing decisional capacity for clinical research or treatment: a review of instruments. Am Psychiatry 2006; 163: 1323-34.

23 Appelbaum PS, Grisso T. The MacArthur Treatment Competence Study. I: mental illness and competence to consent to treatment. Law Hum Behav 1995; 19 : 105-26.

24 Grisso T, Appelbaum PS, Mulvey EP, Fletcher K. The MacArthur Treatment Competence Study. II: measures of abilities related to competence to consent to treatment. Law Hum Behav 1995; 19: 127-48.

25 Candia PC, Barba AC. Mental capacity and consent to treatment in psychiatric patients. Curr Opin Psychiatry 2011; 24: 442-6.

26 Medical Council Ireland. Guide to Professional Conduct and Ethics for Registered Medical Practitioners. Medical Council Ireland, 2009.

27 UN General Assembly. Convention on the Rights of Persons with Disabilities, 2006 A/RES/61/106, Annex I (http://www.refworld.org/docid/4680cd212.html)

28 American Psychiatric Association. Diagnostic and Statistical Manual of Mental Disorders, Fourth Edition, Text Revision (DSM-IV-TR). APA, 2000.

29 National Institute for Health and Care Excellence. Depression in Adults: Recognition and Management (CG90). NICE, 2009 (https://www.nice.org.uk/guidance/cg90).

30 National Institute for Health and Care Excellence. Bipolar Disorder: Assessment and Management (CG185). NICE, 2006 (https://www.nice.org.uk/guidance/cg185).

31 Kay SR, Fiszbein A, Opler LA. The positive and negative syndrome scale (PANSS) for schizophrenia. Schizophr Bull 1987; 13: 261-76.

32 Roth L, Lidz C, Meisel A, Soloff P, Kaufman K, Spiker D, et al. Competency to decide about treatment or research: an overview of some empirical data. Int J Law Psychiatry 1982; 5: 29-50.

33 Roth L, Meisel A, Lidz C. Tests of competency to consent to treatment. Am J Psychiatry 1977; 134: 279-84.

34 Leucht S, Kane JM, Kissling W, Hamann J, Etschel E, Engel RR. What does the PANSS mean? Schizophr Res 2005; 79: 231-8.

35 Howe V, Foister K, Jenkins K. Competence to give informed consent in acute psychosis is associated with symptoms rather than diagnosis. Schizophr Res 2005; 77: 211-4.

36 Carpenter WTJ, Gold JM, Lahti AC. Decisional capacity for informed consent in schizophrenia research. Arch Gen Psychiatry 2000; 57: 533-8.

37 Palmer BW, Dunn LB, Appelbaum PS, Jeste DV. Correlates of treatment- related decision-making capacity among middle-aged and older patients with schizophrenia. Arch Gen Psychiatry 2004; 61: 230-6.

38 Palmer BW, Savla GN. The association of specific neuropsychological deficits with capacity to consent to research or treatment. J Int Neurospychol Soc 2007; 13: $1047-59$.

39 O'Reilly K, Donohoe G, Coyle C, O'Sullivan D, Rowe A, Losty M, et al. Prospective cohort study of the relationship between neuro-cognition, social cognition and violence in forensic patients with schizophrenia and schizoaffective disorder. BMC Psychiatry 2015; 10: 155 